\title{
Velocidad máxima del flujo de la arteria pulmonar fetal en el tercer trimestre de la gestación.
}

Maximum flow velocity of the fetal pulmonary artery in the third trimester of gestation.

Marcos Rubén Rojas Barreto (D) Carlos Garcia Curda (D) Marisol Garcia (D)

https://doi.org/10.54139/salus.v25i2.59

RESUMEN

Introducción: El ultrasonido Doppler ha permitido una mejor comprensión de los cambios que ocurren en el sector pulmonar fetal. Durante este periodo el lecho pulmonar se caracteriza por ser de alta resistencia, elevada presión y bajo flujo, entendiéndose que a medida que avanza la edad gestacional, el flujo hacia el pulmón aumenta por un descenso paulatino de la resistencia pulmonar; estableciéndose como objetivo de esta investigación determinar la velocidad máxima del flujo de la arteria pulmonar fetal en el tercer trimestre de la gestación.

Métodos: Se realizo estudio de diseño transversal, con 475 pacientes, edad gestacional comprendida entre 27 y 41 semanas, atendidas entre septiembre de 2018 y septiembre de 2019, en las cuales a través de Doppler pulsado se obtuvo onda de velocidad de flujo de arteria pulmonar y se calculó su amplitud.

Resultados: Se observó una relación funcional entre las variables, expresada a través de la ecuación de la línea recta, con un ajuste casi perfecto y una asociación inversamente proporcional, estadísticamente significativa $(P<0,001)$.

Conclusión: La evaluación Doppler de la velocidad máxima del flujo de la arteria pulmonar fetal, siendo fácilmente reproducible a través de la evaluación ultrasonográfica, permite establecer valores de referencia en función de la edad gestacional.

Palabras clave: Arteria pulmonar fetal, ecocardiografía fetal, tracto de salida ventrículo derecho.

Unidad de Perinatología. Universidad de Carabobo. Hospital Materno Infantil "Dr. José María Vargas" (Maternidad del Sur) Municipio Valencia del estado Carabobo Venezuela

Autor de correspondencia: Marcos Rubén Rojas Barreto (ID

E-mail: marcosroj96@hotmail.com

Recibido: 02-06-2021

Aprobado: 23-08-2021
Introduction: Doppler ultrasound has allowed a better understanding of the changes that occur in the fetal lung sector. During this period, the lung bed is characterized by being of high resistance, high pressure and low flow, it being understood that as gestational age advances, the flow towards the lung increases due to a gradual decrease in pulmonary resistance, establishing itself as the objective of this Research to determine the maximum velocity of the fetal pulmonary artery in the third trimester of gestation.

Methods: A cross-sectional design study was carried out, with 475 patients, gestational age between 27 and 41 weeks, attended between September 2018 and September 2019, in which pulsed Doppler obtained a pulmonary artery flow velocity wave and its amplitude was calculated.

Results: A functional relationship was observed between the variables, expressed through the equation of the straight line, with an almost perfect fit and an inversely proportional association, being statistically significant $(P<0.001)$.

Conclusion: The Doppler evaluation of the maximum velocity of the fetal pulmonary artery, being easily reproducible through ultrasonographic evaluation, allows establishing reference values based on gestational age.

Keywords: Fetal pulmonary artery, fetal echocardiography, right ventricular outflow tract.

\section{INTRODUCCIÓN}

Durante el desarrollo embrionario el arco aórtico pulmonar, o sexto arco aórtico, origina una rama importante que crece hacia la yema pulmonar en desarrollo. En el lado derecho, la parte proximal se transforma en segmento proximal de la arteria pulmonar derecha y la porción distal desaparece. En el lado izquierdo, la parte distal persiste durante la vida intrauterina en forma de conducto arterial o ducto arterioso (1). Anatómicamente, la arteria pulmonar sale a través de un infundíbulo del ventrículo derecho de forma muy central y se dirige hacia atrás y ligeramente a la izquierda, cruza perpendicular por encima de la aorta y se divide en sus dos ramas pequeñas, derecha e izquierda, que transcurren en dirección lateral hacia ambos pulmones; y se continua con el ductus arterioso, que conecta la arteria pulmonar con la aorta descendente (2).

El árbol vascular fetal es similar al del adulto. No obstante, la presencia de 3 shunts o derivaciones ausentes en el adulto, confiere a la hemodinamia circulatoria fetal características muy particulares (3). Durante este periodo, la sangre 
oxigenada y desoxigenada, inevitablemente se comunican y se mezclan a través de la ubicación estratégica de estos shunts, implicando que la circulación fetal discurra en paralelo, sustentada conjuntamente por ambos ventrículos. El paso de la circulación fetal en paralelo a la circulación posnatal en serie requiere del cierre de los shunts y de la aparición de la oxigenación pulmonar en la vida extrauterina (4).

La oxigenación de la sangre fetal tiene lugar en la placenta (5) desde donde llegan al feto nutrientes y oxígeno a través de la vena umbilical. Parte de la sangre que transporta esta vena alcanza directamente la aurícula derecha a través del conducto venoso, siendo impulsada hacia la aurícula izquierda atravesando el agujero oval. Una pequeña parte de esta sangre rica en oxigeno choca con el septum secundum y se mantiene en la aurícula derecha, donde se mezcla con sangre menos oxigenada procedente de las venas cava superior e inferior.

De este modo, la sangre que llega al ventrículo derecho presenta un grado medio de saturación de oxígeno (6). El circuito arteria pulmonar - ductus arterioso es un territorio de altísimo flujo porque a su través viaja toda la sangre eyectada por el ventrículo derecho, que es el ventrículo dominante en la vida intrauterina, movilizando $2 / 3$ del gasto cardiaco combinado. La mayor proporción del flujo (alrededor del 96\%) eyectado por el ventrículo derecho toma la vía preferencial del ductus arterioso, mientras que el territorio pulmonar solo recibe el $4 \%$ restante, dadas sus características de elevadísima resistencia (7).

Hasta el séptimo mes de vida prenatal los bronquiolos se dividen sin interrupción en canales más numerosos y pequeños y el suministro vascular aumenta de manera constante. Estos canales llegan a su fin en sacos terminales, los cuales aumentan de forma regular durante los 2 últimos meses de vida prenatal y durante varios años de la vida postnatal.

Antes del nacimiento los pulmones están llenos de líquido gracias a la aspiración de líquido amniótico provocado por los movimientos respiratorios presentes durante la vida intrauterina, haciéndose más frecuentemente en etapas tardías de la gestación, estimulando el desarrollo de los pulmones y el acondicionamiento de los músculos respiratorios.

Cuando se inicia la respiración en el nacimiento, los capilares sanguíneos y linfáticos reabsorben la mayor parte del líquido pulmonar, permitiendo el depósito de surfactante en forma de una delgada capa fosfolipídica sobre las membranas celulares alveolares, dando paso a la expansión y llenado de aire de los pulmones (8),

El lecho pulmonar se caracteriza por ser de alta resistencia, elevada presión y bajo flujo y esta fisiología pulmonar fetal permite que a medida que avanza la edad gestacional, el flujo hacia el pulmón aumenta por un descenso paulatino de la resistencia pulmonar (7).

La visualización del tracto de salida del ventrículo derecho se considera parte integral en la evaluación cardiaca fetal, aumentando la tasa de detección de malformaciones cardiacas mayores por encima de las alcanzables solo con la vista de cuatro cámaras. Considerando que $1 \%$ de los embarazos presenta una cardiopatía fetal y la mitad de las cuales se consideran cardiopatías mayores. A pesar de ello, todavía existe una mortalidad global superior al $15 \%$, representando un gran reto en el control del embarazo, siendo la tasa de detección en el segundo trimestre entre 25 y $40 \%$ (9).

Técnicamente, durante la valoración ecográfica cardiaca fetal, se puede realizar un barrido transversal con movimiento cefálico del transductor desde el abdomen fetal a través de la vista de cuatro cámaras y hacia el mediastino superior hasta alcanzar la vista del tracto de salida del ventrículo derecho, corroborando la presencia de un gran vaso que se origina del ventrículo morfológicamente derecho, siendo en condiciones normales la arteria pulmonar, que durante la vida fetal suele ser un poco más grande que la raíz aortica, la válvula pulmonar debe moverse libremente, sin engrosamiento aparente, confirmándose como la arteria pulmonar solo si se ramifica después de un corto trayecto, continuando distalmente hacia el lado izquierdo en el ductus arterioso $(10,11)$.

Para el estudio Doppler, normalmente el perfil de onda de flujo de la arteria pulmonar fetal no debe exceder los 120 $\mathrm{cm} / \mathrm{s}$ (12). Un aumento de dicha velocidad sugiere que la arteria pulmonar recibe menos sangre que lo normal, principalmente en presencia de estenosis o atresia pulmonar, estenosis valvular, o en forma alternativa reducción de flujo sanguíneo secundario a una obstrucción de la válvula tricúspide. Contrario a ello, si el flujo sanguíneo es excesivo, existirá una disminución en su velocidad característico en el síndrome de válvula pulmonar (13).

El estudio ultrasonográfico Doppler de la arteria pulmonar ha permitido la monitorización de la presión arterial pulmonar fetal a través del uso de la relación tiempo de aceleración/ tiempo de eyección (14) y otras investigaciones han determinado que esta relación aumenta a medida que avanza la edad gestacional, aproximándose al diagnóstico de madurez pulmonar fetal (15-17).

Las características de las ondas de velocidad de flujo Doppler en la arteria pulmonar y su relación con la edad gestacional fueron determinadas por Laudy et al. (18). Herren et al. (19), establecieron rangos de referencia de índice de pulsatilidad y velocidad sistólica máxima de los segmentos proximal, medial y distal de la arteria pulmonar fetal entre 19 y 39 semanas de gestación. Guerrero (20) analizó la velocidad máxima de onda de flujo de la arteria pulmonar fetal antes y después de la administración intramuscular de betametasona como inductor de madurez pulmonar fetal. 
La introducción del ultrasonido Doppler ha permitido una mejor comprensión de los cambios que ocurren en el sector pulmonar fetal, así como, a través de los avances en cirugía cardiaca pediátrica, en los últimos 30 años se han producido mejoras espectaculares en el pronóstico de las cardiopatías congénitas. (9) Mediante esta técnica se ha podido establecer que a medida que avanza la edad gestacional, la fracción destinada a perfundir el árbol pulmonar se va incrementando conforme avanza el proceso de crecimiento y desarrollo del pulmón, el cual pasa por una serie de etapas que tienen por finalidad incrementar la arborización de bronquios preterminales y terminales, la multiplicación de los alveolos y en consecuencia el incremento de la red perialveolar responsable final de la hematosis (7).

Nuestra intención fue determinar la variación de la velocidad de la onda espectral de la arteria pulmonar y su relación con la edad gestacional para establecer valores de referencia que se puedan emplear en la evaluación perinatal, por lo que se planteó como objetivo general de esta investigación determinar la velocidad máxima del flujo de la arteria pulmonar fetal en el tercer trimestre de la gestación.

\section{MATERIALES Y MÉTODOS}

Se realizó un estudio de diseño transversal en una población caracterizada por gestantes que acudieron a la unidad de Perinatología del Hospital Materno-Infantil Dr. José María Vargas, en Valencia, Edo. Carabobo, y cuya muestra estuvo constituida por 475 pacientes con edad gestacional comprendida entre 27 y 41 semanas, atendidas entre septiembre de 2018 y septiembre de 2019. Previo consentimiento informado de las pacientes que aceptaron su inclusión en la muestra y aprobado por el comité de ética de la institución

Los criterios de inclusión comprendieron pacientes en tercer trimestre de la gestación, sin comorbilidades asociadas, y gestación simple con feto normal. Los criterios de exclusión fueron gestaciones en primer y segundo trimestre, gestación múltiple, fetos con malformaciones estructurales evidentes o restricción de crecimiento y pacientes con comorbilidades.
Según recomendaciones estandarizadas, se realizó ecocardiografía fetal con equipo de ultrasonido marca Esaote MyLab 70 XVision con transductor convex multifrecuencial (3,5 a $5 \mathrm{MHz}$ ), empleando la vía transabdominal. Partiendo del corte de cuatro cámaras, se deslizó el transductor hacia la cabeza fetal hasta obtener el cruce normal de la aorta y la arteria pulmonar principal en sus orígenes.

Alternativamente, se empleó la técnica rotacional o de rotación, donde desde el corte de cuatro cámaras, se roto el transductor hacia el hombro derecho del feto. Con ambas técnicas, una vez que se obtuvo el corte de tracto de salida de ventrículo izquierdo, se angula el transductor en sentido cefálico hasta que se observó la arteria pulmonar con una dirección casi perpendicular a la de la aorta (10, 21). La arteria pulmonar se origina en la parte más central del ventrículo derecho demostrando la existencia de concordancia ventrículo-arterial y luego de cruzar la aorta se divide en sus 2 ramas (Fig. 1) y en el ductus arterioso, que aparece como la prolongación del vaso principal (12).

Para el estudio Doppler se colocó el volumen de muestra adaptado al calibre del vaso justo a continuación de la válvula pulmonar con ángulo de insonación cercano a $0^{\circ}$ obteniendo el perfil de onda (Fig. 2) y calculándose el valor de la velocidad máxima al medir la amplitud de la onda de flujo (12). La velocidad en el tronco pulmonar principal aumenta rápidamente después del inicio de la eyección para alcanzar su pico durante la sístole. Luego cae, pero la disminución en la velocidad se interrumpe y con frecuencia se observa una incisura definida en el perfil de velocidad 22).

Los datos fueron plasmados en una hoja de Excel $\circledast$, el análisis estadístico se realizó con el software libre Past 3.24 y se calculó la distribución percentilar para cada edad gestacional representándose en tablas. Seguidamente se realizó el análisis de regresión y análisis de correlación para establecer el grado de asociación entre las variables.

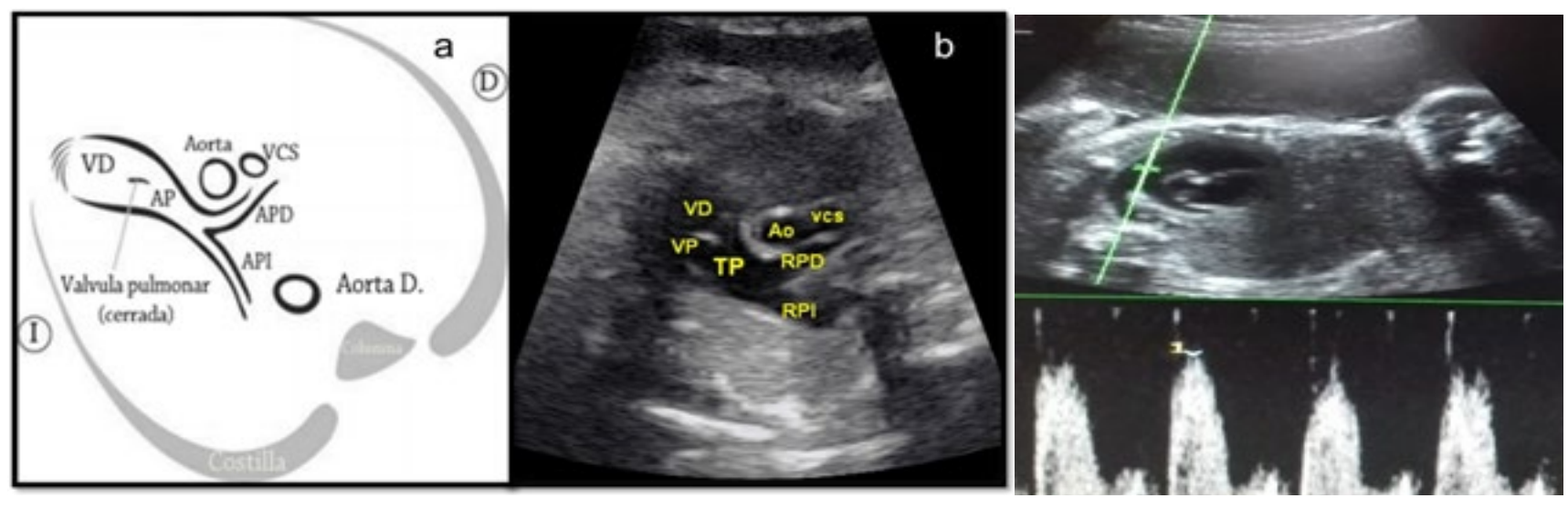

Fig. 1. a). Ilustración de tracto de salida del ventrículo derecho. Tomado de Guías prácticas ISUOG (actualizada 2017): evaluación ecográfica de tamizaje del corazón fetal. b). Ecofotograma de arteria pulmonar fetal bifurcándose en arteria pulmonar derecha e izquierda. VD: ventrículo derecho. VP: válvula pulmonar. TP: tronco pulmonar. RPD: rama pulmonar derecha. RPI: rama pulmonar izquierda. Ao: Aorta. VCS: vena cava superior. c) Ecofotograma donde se visualiza el volumen de la muestra en la arteria pulmonar fetal por encima de la válvula pulmonar y la onda espectral) Rojas, Marcos. (2018)

\section{Salus}




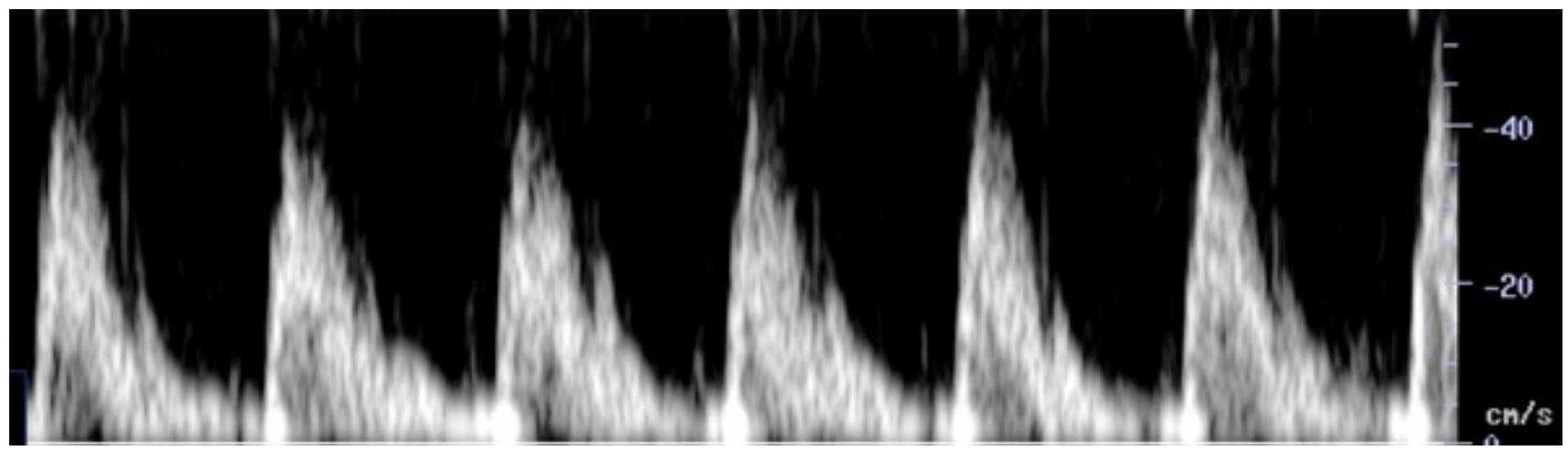

Figura 2. Onda espectral Doppler de arteria pulmonar fetal. Rojas, Marcos. (2018).

\section{RESULTADOS Y DISCUSIÓN}

Durante el estudio se obtuvo los valores de la velocidad máxima de la onda de flujo de la arteria pulmonar fetal en la totalidad de la muestra. En la tabla 1 se presenta los rangos de normalidad de la velocidad máxima de la onda de flujo de la arteria pulmonar fetal en pacientes con gestación simple comprendida entre 27 y 41 semanas.

En la fig. 3 se aprecia gráficamente la ecuación predictiva de la velocidad máxima de la onda de flujo de la arteria pulmonar fetal en función de la edad gestacional y la relación lineal entre ambas variables.

Se observa una relación funcional entre las variables, expresada a través de la ecuación de regresión lineal simple para predecir el valor de la velocidad máxima de la onda de flujo de la arteria pulmonar fetal de acuerdo con la edad gestacional, resultando: Y (Velocidad máxima de la arteria pulmonar fetal $)=-3,8546 * X($ Edad gestacional en semanas) $+196,82$.
Tabla 1. Distribución percentilar por edad gestacional de la velocidad máxima de la arteria pulmonar fetal.

\begin{tabular}{|c|c|c|c|c|}
\hline \multicolumn{4}{|c|}{ Velocidad máxima de arteria pulmonar fetal (cm/s) } \\
\hline $\begin{array}{c}\text { Edad Gestacional } \\
\text { (semanas) }\end{array}$ & $n$ & $\mathrm{P} 10$ & $\mathrm{P} 50$ & $\mathrm{P} 90$ \\
\hline 27 & 36 & 79,5 & 89,6 & 95,8 \\
\hline 28 & 33 & 76,2 & 88,4 & 94,4 \\
\hline 29 & 35 & 80,1 & 85,6 & 90,4 \\
\hline 30 & 32 & 73,2 & 79,8 & 90,8 \\
\hline 31 & 32 & 76,5 & 81,6 & 91,4 \\
\hline 32 & 31 & 66,3 & 75,6 & 80,0 \\
\hline 33 & 31 & 67,7 & 72,5 & 76,6 \\
\hline 34 & 31 & 64,4 & 68,9 & 74,1 \\
\hline 35 & 30 & 61,2 & 65,6 & 70,0 \\
\hline 36 & 34 & 56,6 & 60,2 & 64 \\
\hline 37 & 35 & 50,2 & 53,5 & 60,2 \\
\hline 38 & 34 & 43,0 & 47,0 & 50,0 \\
\hline 39 & 30 & 39,9 & 44,1 & 50,2 \\
\hline 40 & 30 & 38,1 & 39,9 & 43,1 \\
\hline 41 & 21 & 39,1 & 39,6 & 40,7 \\
\hline
\end{tabular}

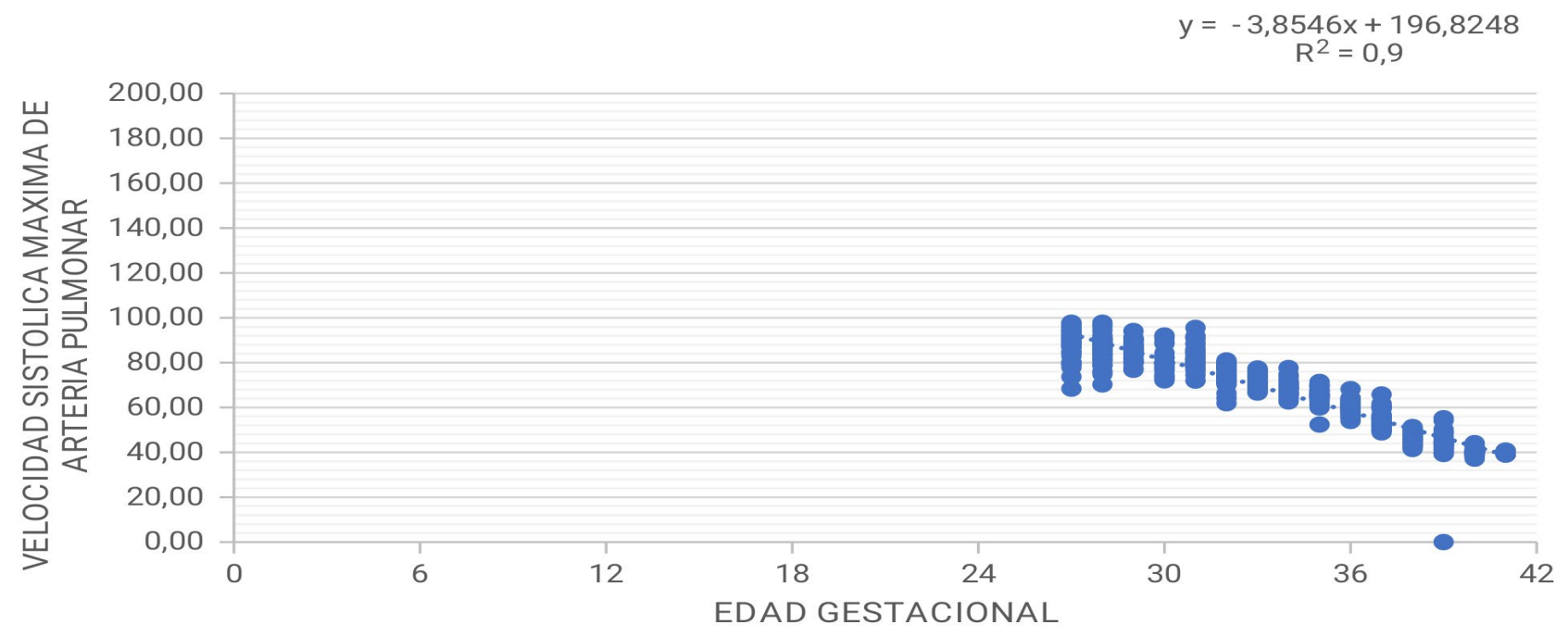

Fig. 3. Velocidad máxima de la onda de flujo de la arteria pulmonar fetal expresada en $\mathrm{cm} / \mathrm{s}$, en función de la edad gestacional expresada en semanas. 
El coeficiente de determinación $\left(R^{2}\right)$ para esta ecuación fue: 0,90 , demostrando un ajuste de $90 \%$ en la velocidad máxima de la onda de flujo de la arteria pulmonar fetal al variar la edad gestacional. Al determinar el grado de asociación entre las variables, el valor del coeficiente de correlación de Pearson ( $r$ ) entre las mismas fue: $-0,95$ expresando una correlación inversa o negativa, donde se establece una asociación inversamente proporcional manifestando que a medida que aumenta la edad gestacional disminuye la velocidad máxima del flujo de la arteria pulmonar fetal en la misma proporción. Como prueba de significación se obtuvo un valor de $\mathrm{t}: 70,283$, siendo mayor que el valor critico de t para $\alpha=0,001$, afirmando una relación estadísticamente significativa. Por tanto, en este estudio, la velocidad máxima de la onda de flujo de la arteria pulmonar fetal y la edad gestacional están linealmente relacionados $(P<0,001)$.

Después del parto la absorción de oxígeno y la eliminación de dióxido de carbono del cuerpo se produce en los pulmones. Se absorben sustratos energéticos en el tracto gastrointestinal y pasan al hígado a través del sistema venoso portal antes de entrar en la circulación general. En el feto, la absorción de oxígeno y la eliminación de dióxido de carbono se realiza en la placenta a través de la circulación umbilical. Los sustratos energéticos son transportados desde la circulación materna a través de la membrana placentaria y se transfieren al cuerpo fetal a través de la vena umbilical. Aunque una parte de los sustratos entran a la circulación hepática, una cantidad variable pasa por alto el hígado para entrar en la circulación fetal general a través del conducto venoso mezclándose con la sangre sistémica venosa antes de entrar en los ventrículos cardíacos para ser expulsada y perfundir el cuerpo fetal (22).

El flujo sanguíneo pulmonar aumenta considerablemente durante la gestación, siendo aproximadamente el 12\% del gasto cardiaco combinado a las 20 semanas, y del $25 \%$ después de las 30 semanas. Los mecanismos por los cuales se reduce la resistencia vascular pulmonar fetal sugieren que la expansión progresiva de los pulmones estimula la producción de prostaglandinas, siendo la prostaciclina el principal vasodilatador pulmonar. Durante el nacimiento se considera la posibilidad de que eliminación de líquido presente en las vías respiratorias fetales y el reemplazo por gas desarrolla una presión intrapleural negativa, con un gradiente desde las vías respiratorias hacia afuera hasta la pleura, y esto tiende a dilatar los vasos pulmonares (22).

El efecto Doppler permite medir muchas variables asociadas con la hemodinamia fetal (23), permitiendo el estudio del flujo sanguíneo de forma inocua y reproducible (24), siendo la evaluación de la velocidad de flujo de la arteria pulmonar un método de estimación no invasivo durante la vida fetal.

El flujo sanguíneo a través de los pulmones es bajo durante la vida fetal, lo que se ha explicado sobre la base de una alta resistencia vascular pulmonar, siendo demostrado por Laudy et al. (18), en el segundo trimestre de la gestación donde la arteria pulmonar fetal muestra un patrón único de forma de onda Doppler caracterizado por una fase de aceleración rápida del flujo inicial y una fase de desaceleración igualmente rápida, produciendo un pico sistólico en forma de aguja pero sin variaciones estadísticamente significativas en la velocidad contrario a lo reportado en la presente investigación, donde se observan cambios graduales en la forma de la onda Doppler con una reducción estadísticamente significativa de la velocidad máxima de la arteria pulmonar fetal asociado a la disminución de la resistencia vascular pulmonar en el tercer trimestre y relacionado linealmente con la edad gestacional.

Herren et al. (19), describieron resultados similares a esta investigación al observar diferencias estadísticamente significativas en la velocidad pico de la arteria pulmonar fetal correlacionadas con la edad gestacional y representadas mediante ecuaciones lineales logrando determinar con éxito los rangos de referencia entre 19 y 39 semanas de gestación.

Finalmente, Guerrero (20), no encontró diferencias estadísticamente significativas en la velocidad de la arteria pulmonar fetal atribuible a la administración de betametasona en el tercer trimestre de la gestación, más que los cambios observados en este estudio propios de su correlación con la edad gestacional.

\section{CONCLUSIÓN}

La determinación de una relación inversamente proporcional entre la velocidad máxima del flujo de la arteria pulmonar fetal y la edad gestacional permiten establecer valores de referencia que pueden ser utilizados durante la evaluación perinatal con el fin de identificar cambios en la circulación fetal consecuencia de alteraciones cardiacas estructurales a nivel cono troncal.

El empleo de Doppler permite evaluar a la arteria pulmonar fetal, con la finalidad de intuir los cambios propios de la edad gestacional en el territorio vascular pulmonar, al discriminar las modificaciones hemodinámicas de madurez pulmonar fetal debido a la disminución progresiva de la resistencia vascular en el tercer trimestre de la gestación.

A pesar de que la medida de la onda de flujo de la velocidad de la arteria pulmonar fetal es sencilla de obtener y de fácil reproducción mediante ultrasonografía, se debe tener experiencia en la evaluación de la misma, basado en recomendaciones establecidas, para obtener resultados precisos que ayuden a establecer conductas obstétricas acertadas.

\section{REFERENCIAS BIBLIOGRÁFICAS}

1. Sadler T. Sistema cardiovascular. In Langman Embriología Médica. 12th ed. Barcelona: Wolters Kluwer; 2012. p. 162-200.

2. Martinez J, del Rio M, Bennasar M, Hernandez E, Gratacos E. Exploracion anatomica cardiaca: ecocardiografia fetal. In Gratacos E, Gomez R, Romero R, Nicolaides K, Cabero L. Medicina Fetal. Madrid: Editorial Medica Panamericana; 2009. p. 317-327. 
3. Cafici D. Circulacion fetal en condiciones normales y en respuesta a la hipoxia. En: Ultrasonografia Doppler en Obstetricia. Buenos Aires: Ediciones journal; 2008. p. 227-252.

4. Herraiz I, Gomez E, Galindo A. Circulacion fetal y neonatal. En: Galindo A, Gratacos E, Martinez J. Cardiologia Fetal. Madrid: Marban; 2015. p. 38-43.

5. Beckman C, Ling F, herbert W, Laube D, Smith R. Fisiologia maternofetal. En: Obstetricia y Ginecologia. 8th ed. Barcelona: Wolters Kluwer; 2019. p. 121-151.

6. Nebot J. Embriología del corazón y circulación fetal. En: Gratacos E, Gomez R, Romero R, Nicolaides K, Cabero L. Medicina Fetal. Madrid: Editorial medica panamericana; 2009. p. 309-315.

7. Medina A. Circuito Arteria Pulmonar-Ductus arterioso. Ultrasonografia Embriofetal. 2010; 5(1): 84-90.

8. Sadler T. Sistema respiratorio. IEn: Langman Embriología Médica. 12a Ed. Barcelona: Wolters Kluwer; 2012. p. 201-207.

9. Gratacos E, Galindo A, Martinez J. Concepto y ambito de la cardiologia fetal. In Galindo A, Gratacos E, Martinez J. Cardiologia fetal. Madrid: Marban; 2015. p. 1-6.

10. Carvalho J, Allan L, Chaoui R, Copel J, DeVore G, Hecher $\mathrm{K}$, et al. ISUOG practice guidelines (updated): sonographic screening examination of the fetal heart. Ultrasound Obstet Gynecol. 2013; 41: 348-359.

11. Moon A, Donofrio M, Cohen S, Yagel S. Indications for fetal echocardiography: Screening in low - and high- risk populations. En Yagel S, Silverman N, Gembruch U. Fetal Cardiology Embryology, Genetics, Physiology, Echocardiographic evaluation, Diagnosis, and Perinatal management of cardiac diseases. 3rd ed. New York: Taylor and Francys Group, LLC; 2019. p. $86-100$.

12. Puente J, Gomez E, Galindo A. Exploracion cardiaca normal. In Galindo A, Gratacos E, Martinez J. Cardiologia Fetal. Madrid: MARBAN; 2015. p. 66-86.

13. Allan L, Cook A, Huggon I. Grandes arterias y arcos: normales y anormales. En: Ecocardiografia fetal una guia practica. 1a Ed. Buenos Aires: Ediciones Journal; 2010. p. 76-118.

14. Hong-Yan Z, Feng-Qin X, Chuan-Xi L, Gang Z. Clinical applicability of monitoring pulmonary artery blood flow acceleration time variations inmonitoring fetal pulmonary artery pressure. Adv Clin Exp Med 2018; 27(12): 1723-1727.

15. Huaman M, Campodonico L, Garcia N, Pacheco J. Doppler del tronco de la arteria Pulmonar en la prediccion de la madurez pulmonar fetal. Revista Peruana de Ginecologia y Obstetricia. 2009; 55: 120-125.

16. Azpurua H, Norwitz E, Campbell K, Funai E, Pettker C, Kleine $\mathrm{M}$, et al. Acceleration/ejection time ratio in the fetal pulmonary artery predicts fetal lung maturity. AmJ ObstGynecol 2010; 203(1):40.e1-8. doi: 10.1016/j.ajog.2010.01.075.

17. Peñaherrera M, Villalobos N, Lopez O, Lopez A, Rodriguez V, Valero N. Tiempo de aceleracion y tiempo de eyeccion de la arteria pulmonar fetal como predictor de madurez pulmonar. Dom. Cien. 2019; 5(3): 42-56.
18. Laudy J, De Ridder M, Wladimiroff J. Doppler velocimetry in branch pulmonary arteries of normal human fetuses during the second half of gestation. Pediatr Res 1997; 41: 897-901.

19. Herren H, Araujo E, Martins, Carvalho R, Marcolin A, Da Silva $\mathrm{F}$, et al. Reference ranges of Doppler parameters of foetal pulmonary artery segments between 19 and 39 weeks of gestation. J Matern Fetal Neonatal Med. 2016; 29(1): 85-90.

20. Guerrero R. Velocidad maxima de la arteria pulmonar fetal antes y despues del uso de betametasona en embarazos entre 28 y 34 semanas en el servicio de perinatologia del hospital "Dr. Adolfo Prince Lara". Tesis especializacion; 2017.

21. Drose J. Exploracion: indicaciones y tecnica. En: Drose J. Ecocardiografia fetal. 2da. Ed. Amolca; 2011. p. 15-72.

22. Rudolph A. Circulation in the normal fetus and cardiovascular adaptations to birth. En: Yagel S, Silverman N, Gembruch U. Fetal Cardiology Embryology, Genetics, Physiology, Echocardiographic Evaluation, Diagnosis, and Perinatal Management of Cardiac Diseases. 3rd ed.: Taylor \& Francis Group, LLC; 2019. p. 101-119.

23. Sosa A, Zurita J, Schenone C, Schenone M, Prieto F. Doppler evaluation of the fetal pulmonary artery pressure. J Perinatal Med 2019; 47(2): 218-221.

24. Agostini M, Moran R, Cordano C, Garat F. Utilidad de la ecografía y del eco-Doppler color en pacientes de alto riesgo obstétrico. Rev Urug Med Int 2021; 6(2): 67-71. 